\title{
Fairness Criteria for Algebraic Curves
}

\author{
Pavel Chalmovianský, Bert Jüttler
}

July 19, 2004

\begin{abstract}
We develop methods for the variational design of algebraic curves. Our approach is based on truly geometric fairness criteria, such as the elastic bending energy. In addition, we take certain feasibility criteria for the algebraic curve segment into account. We describe a computational technique for the variational design of algebraic curves, using an SQP (sequential quadratic programming) - type method for constrained optimization. As demonstrated in this paper, the powerful techniques of variational design can be used not only for parametric representations, but also for curves in implicit form.
\end{abstract}

Keywords. Variational design, algebraic curves, implicit representation.

\section{Introduction}

For parametric curves and surfaces, the various techniques of variational design are now widely being used in geometric modeling $[2,5,6]$. These techniques have been highly successful in various applications, including scattered data fitting (e.g. for reverse engineering), where they help to generate high-quality curves and surfaces from measurement data (point clouds).

Implicitly defined curves and surfaces have several potential advantages. For instance, surface-surface intersections can easily be traced if one surface is given in implicit form, and the other surface by a parametric representation. Also, the algorithms for curve and surface fitting do not assume the existence of a parameterization of the data, which is often difficult to generate, in particular for more complex data sets (see [8,9] and the references cited therein). 
Variational design of curves and surfaces in implicit representation, however, has not attracted much attention so far. Similar to the parametric case, such techniques are needed in order to improve the quality of the results.

This is closely related to level set methods which use geometric criteria for controlling the evolution of a curve (or surface) [12]. Typically, these techniques are based on the local behavior of a curve (e.g. depending on curvature).

Based on globally defined, truly geometric fairness criteria, such as the elastic bending energy (cf. [3]), we discuss a method for the variational design of algebraic curves. In addition to the fairness measures, our objective function also takes certain feasibility criteria for the algebraic curve segment into account. We develop a computational technique for the variational design of algebraic curves, using an SQP-type method for constrained optimization.

\section{Fairness Functionals for Planar Curves}

Following the notations introduced in [11], we use the Bernstein-Bézier representation of the algebraic curve segment with respect to a fixed basis triangle $\triangle \mathbf{v}_{0} \mathbf{v}_{1} \mathbf{v}_{2} \subset \mathbb{R}^{2}$. Throughout this paper, we consider a segment of an algebraic curve of order $n$. This segment is a part of the zero contour of a polynomial $G$ of degree $n$,

$$
\tilde{\mathcal{C}}=\{\mathbf{p} \mid G(\mathbf{p})=0 \text { and } \mathbf{p} \in D\}
$$

where the domain $D$ (which is to satisfy $\triangle \mathbf{v}_{0} \mathbf{v}_{1} \mathbf{v}_{2} \subset D$ ) is the strip bounded by two parallels through $\mathbf{v}_{0}, \mathbf{v}_{1}$ with the direction of a suitable unit vector $\overrightarrow{\mathbf{r}}=\left(r_{1}, r_{2}\right)$. The end points of the curve segment are the vertices $\mathbf{v}_{0}$ and $\mathbf{v}_{1}$, and the lines $\overline{\mathbf{v}_{0} \mathbf{v}_{2}}, \overline{\mathbf{v}_{1} \mathbf{v}_{2}}$ of the domain triangle are assumed to be the tangents at these two points.

In order to obtain curves which are useful for geometric modeling, we assume that there exists a curve segment which has the shape of a single $\operatorname{arc} \mathcal{C}$ connecting $\mathbf{v}_{0}$ and $\mathbf{v}_{1}$, without singularities along this arc, such that $\mathcal{C} \subseteq \tilde{\mathcal{C}}$. Suitable feasibility criteria, which are needed in order to achieve and to maintain this shape during the optimization, will be discussed in Section 3.

We consider $G$ as a linear combination of Bernstein-Bézier polynomials over $\triangle \mathbf{v}_{0} \mathbf{v}_{1} \mathbf{v}_{2}$ with coefficients

$$
\mathbf{b}=\left(b_{\mathbf{i}}\right)_{|\mathbf{i}|=n, \mathbf{i} \in \mathbb{Z}_{+}^{3}} \in \mathbb{R}^{d_{n}} \quad \text { where } \quad d_{n}=\left(\begin{array}{c}
n+2 \\
2
\end{array}\right) .
$$



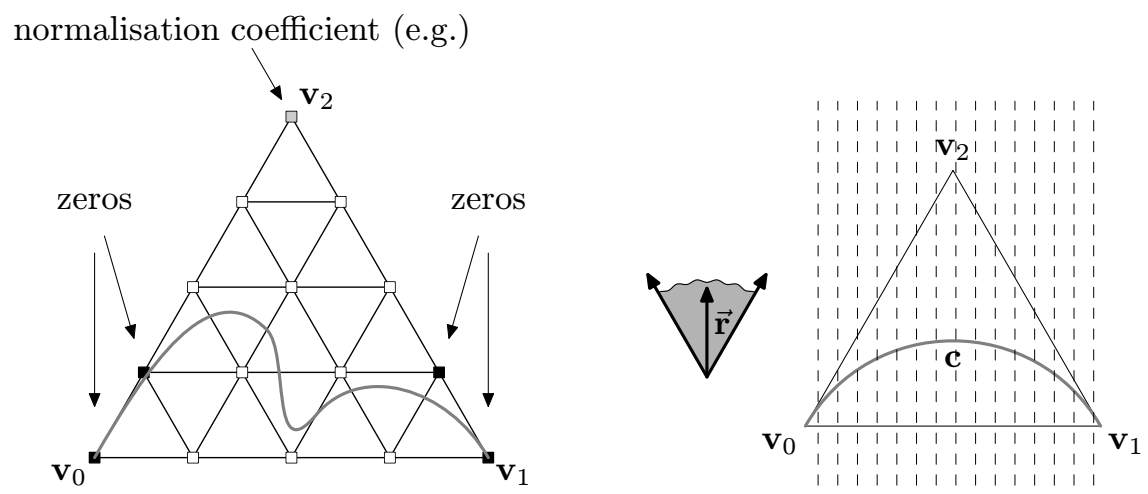

Figure 1: Left: boundary values of Bernstein-Bézier coefficients; right: parameterization of the segment as a graph of a function.

As a necessary condition for the end point and the end tangent property, the Bernstein-Bézier coefficients $b_{\mathbf{i}}$ satisfy

$$
b_{n, 0,0}=b_{n-1,0,1}=b_{0, n, 0}=b_{0, n-1,1}=0,
$$

(see Figure 1, left). These conditions are also sufficient, provided that both segment end points are non-singular.

\subsection{Parameterization}

In order to evaluate the various quantities needed during the optimization process (bending energy and its derivatives), we need a parameterization of the curve segment $\mathcal{C}$. This parametric representation has the form $\mathbf{c}(t)=$ $\left(x_{1}(t), x_{2}(t)\right)$ for $t \in I$, where $I=[0,1]$ is the parameter interval, and

$$
G(\mathbf{c}(t))=0 \quad \text { for } \quad t \in I .
$$

Clearly, such a parameterization is generally not available in closed form; only a numerical approximation can be given.

According to a well-known result from the theory of elastic curves, these curves can turn at most by angle of $\pi$ (see e.g. [3]). Motivated by this fact, we assume that the curve segment $\mathcal{C}$ is a graph of a function, where the " $y$ axis" has an appropriately chosen direction $\overrightarrow{\mathbf{r}}$, such that it is contained in the wedge spanned by the vectors $\mathbf{v}_{2}-\mathbf{v}_{0}$ and $\mathbf{v}_{2}-\mathbf{v}_{1}$. Under this assumption, the parameterization of the algebraic curve can be obtained by projecting 
it parallel to $\overrightarrow{\mathbf{r}}$ onto the edge $\overline{\mathbf{v}_{0} \mathbf{v}_{1}}$ of the basis triangle. Consequently, the parametric representation has the form

$$
\mathbf{c}(t)=\left(x_{1}(t), x_{2}(t)\right)^{\top}=(t, 0)^{\top}+y(t) \overrightarrow{\mathbf{r}}, \quad t \in[0,1],
$$

where $y(t)$ is one of the $n$ roots of the bivariate polynomial $G(\mathbf{p})$ along the line $(t, 0)+\lambda \overrightarrow{\mathbf{r}}$. Clearly, one has to pick the "right" root among the possibly $n$ different ones. For the sake of further computation, we need the function $y(t)$. Since $G$ is a polynomial and since we suppose that no singular points along the curve segment $\mathcal{C}$ exist, the function $y(t)$ is $C^{2}$ in $[0,1]$.

The ordinate $y$ of a point $\mathbf{c}(t)$ depends both on the abscissa $t$ and Bernstein-Bézier coefficients b. During the optimization we will need the derivatives of $y=y(t, \mathbf{b})$ with respect to both quantities. However, the result of the optimization itself depends only on the control coefficients vector $\mathbf{b}$. In order to keep the notations simple, let $F\left(x_{1}, x_{2}, \mathbf{b}\right)$ be a function such that

$$
F\left(x_{1}(t, \mathbf{b}), x_{2}(t, \mathbf{b}), \mathbf{b}\right)=G(\mathbf{c}(t))=0,
$$

see (2),(4) and (5). Similarly,

$$
F_{i}=\partial F / \partial x_{i} \quad \text { and } \quad F_{i j}=\partial^{2} F / \partial x_{i} \partial x_{j} \quad \text { for } \quad i, j \in\{1,2\} .
$$

The proof of the following lemma follows from the implicit function theorem.

Lemma 1 The derivatives of the abscissa $y=y(t, \mathbf{b})$ with respect to the parameter $t$ and with respect to the coefficients in $\mathbf{b}=\left(b_{\mathbf{i}}\right)_{|\mathbf{i}|=n, \mathbf{i} \in \mathbb{Z}_{+}^{3}}$ can be computed by differentiating (6). All these derivatives exist, provided that $F$ is sufficiently often differentiable, and $r_{1} F_{1}+F_{2} \neq 0$.

\subsection{Bending energy and objective function}

We consider the bending energy

$$
f_{\text {bend }}(\mathcal{C})=\int_{\mathcal{C}} \kappa^{2} \mathrm{~d} s
$$

of a planar curve segment, where $\kappa$ and $s$ are the curvature and the arc length parameter. Clearly, this is a geometrically invariant fairness measure, independent of the parameterization.

The curves minimizing (8) are called elastica. There exists no unique minimizer to $G^{1}$ Hermite boundary data. Instead, there exists a sequence of local minima with different lengths. See e.g. [7] for additional information. 
The objective function takes the form

$$
f(\mathbf{b})=f_{\text {bend }}(\mathcal{C})=\int_{\mathcal{C}} \kappa^{2} \mathrm{~d} s=\int_{0}^{1}[\dot{\mathbf{c}}, \ddot{\mathbf{c}}]^{2}\|\dot{\mathbf{c}}\|^{-5} \mathrm{~d} t
$$

where $\left[\overrightarrow{\mathbf{a}}_{1}, \overrightarrow{\mathbf{a}}_{2}\right]=\operatorname{det}\left(\overrightarrow{\mathbf{a}}_{1}, \overrightarrow{\mathbf{a}}_{2}\right)$. We will need the first and second derivatives of $f$ with respect to $b_{i}$ and $t$ during the optimization process. They can be evaluated by applying the differentiation to the function under the integral in (9). For the sake of brevity, we omit the details (see [4]).

To do the optimization, we map any polynomial to the coefficient space. Any polynomial of degree $n$ corresponds to a point $\mathbf{b} \in \mathbb{R}^{d_{n}}$ (see also (2)). Conversely, each point of $\mathbf{b} \in \mathbb{R}^{d_{n}}-\{\overrightarrow{\mathbf{0}}\}$ represents some homogeneous polynomial of degree $n$.

Further properties of the objective function $f$ can be deduced from the boundary conditions for segment $\mathcal{C}$ and the fact that the function $G(\mathbf{p})$ is polynomial. Since four coefficients vanish (see Figure 1, left), it suffices to optimize just in the subspace of $\mathbb{R}^{d_{n}}$ given by (3). Moreover, the objective function is a homogeneous function of order 0 , i.e.

$$
f(k \mathbf{b})=f(\mathbf{b})
$$

for all $k \neq 0$. More precisely, the objective function is geometrically invariant, i.e. it is not changed by multiplying the equation of the algebraic curve by a non-zero constant $k$, since the zero contour does not change.

Due to the homogeneity of the objective function, the coordinates of the point $\mathbf{b}$ (representing the algebraic curve) can be normalized by choosing some of its non-zero coordinates (see Figure 1, left).

\section{Minimization of fairness functionals}

Clearly, the minimum of a highly non-linear function cannot be calculated directly. Hence, we approximate the original function with a sequence of simpler functions, for which the minimum can be calculated. This leads to the sequential quadratic programming (SQP)-type method which is based on a local quadratic approximation.

We introduce the notion of the feasibility for the algebraic curve.

Definition 1 An algebraic curve given by (1) is feasible, if the following three conditions are met 
(i) boundary conditions: It satisfies the prescribed $G^{1}$ boundary conditions at the endpoints

(ii) functionality: The part of the curve between endpoints can be parameterized as in (5) using the chosen direction $\overrightarrow{\mathbf{r}}$

(iii) regularity: The parameterized part of the curve contains no singular point (in the sense of algebraic geometry).

Note that he parameterization (ii) can be assumed to exist since the tangent of an elastic curve turns at most by $\pi$ (see [3]). Even if this condition is not satisfied, the methods described in the remainder of this paper can be extended to the general case, by combining several local parameterizations. ${ }^{1}$

In the sequel, we assume that the initial algebraic curve for the optimization meets the feasibility requirements. Consequently, we have a segment $\mathbf{c}^{0}(t)$ (described by the Bernstein-Bézier coefficient vector $\mathbf{b}^{0}$ over the triangle), which is a graph of a function with respect to the chosen direction $\overrightarrow{\mathbf{r}}$. We are looking for the minimum of $f(\mathbf{b})$ with respect to the control points, such that the feasibility conditions are still satisfied.

The algorithm calculates a sequence of curves represented by coefficients

$$
\mathbf{b}^{0}, \mathbf{b}^{1}, \mathbf{b}^{2}, \ldots, \mathbf{b}^{h}, \ldots \text { for } h \in \mathbb{Z}_{+},
$$

such that the conditions of the Definition 1 are always fulfilled. hence, each curve segment can be parameterized by $\mathbf{c}^{h}(t)$. Moreover, the values of the function $f$ evaluated on the curves converges monotonically to a local minimum.

Algorithm 1 The sequence (11) is generated by the following iteration:

1. Approximate the objective function $f(\mathbf{b})$ by a quadratic function $q^{h}(\mathbf{b})$ in the neighborhood of point $\mathbf{b}^{h}$.

2. Find the extremum $\mathbf{b}^{*}$ of the quadratic function $q^{h}(\mathbf{b})$.

3. Choose the step size $\tau^{h+1} \in \mathbb{R}$ and find the next point from

$$
\mathbf{b}^{h+1}=\left(1-\tau^{h+1}\right) \mathbf{b}^{h}+\tau^{h+1} \mathbf{b}^{*}
$$

such that the feasibility is maintained.

We use Gaussian quadrature to evaluate the integrals during the minimization. The details of the minimization algorithm are omitted due to space limitations, see [4].

\footnotetext{
${ }^{1}$ More precisely, we can always guarantee that such a parameterization exists locally in a neighborhood of a regular point on the curve. Therefore, if the initial curve segment do not satisfy this property, we can split it to several pieces so that they already do.
} 


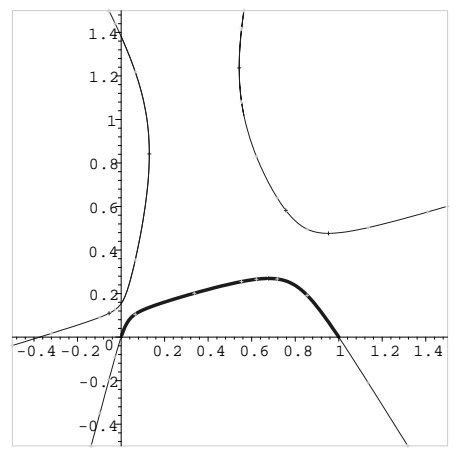

(a)

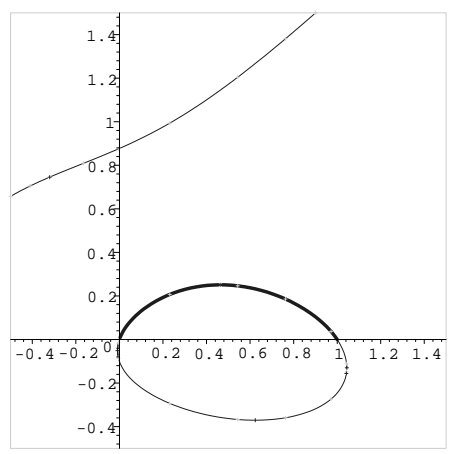

(b)

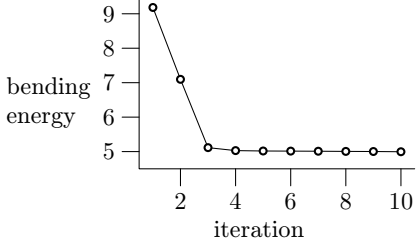

(c)

Figure 2: An optimization of a cubic curve. Bending energy for the thick part of the curves: $f_{\text {bend }}=9.18(\mathrm{a}), f_{\text {bend }}=4.96(\mathrm{~b})$. Convergence of the bending energy to minimum (c).

As a first example, this fairing has been applied to the cubic algebraic curve which is shown in Figure 2, left. The value of the bending energy for the initial curve segment is equal to 9.18. After several iterations we arrived at a curve with energy 4.96. The values of the bending energy for the sequence of curves generated during the fairing are shown in Figure 2 c.

Unfortunately, the solution of a quadratic approximation of the (9) often produces infeasible curves. The curve may not be a function in the direction $\overrightarrow{\mathbf{r}}$ or it may have singularities. We use two different techniques to resolve these problems.

First, in order to maintain the functionality of the curve segment, we restrict the domain of the optimization. This is achieved by choosing the stepsize in each iteration accordingly. More precisely, we use a Runge-Kutta-type method for tracing the candidate curve and use binary search for choosing the correct stepsize. Clearly, this approach keeps the functional shape property.

Second, we use a penalty function for avoiding curves with singularities. It keeps the solutions (11) away from the points in $\mathbb{R} \mathbb{P}^{d_{n}-5}$, which represent curves with singularities along the considered $\operatorname{arc} \mathcal{C}$. Clearly, the additional term in the objective function will cause an increase of the bending energy for the final curve.

To avoid points where both partial derivatives, $G_{1}$ and $G_{2}$ vanish, we 
have chosen the penalty function

$$
f_{\text {penalty }}(\mathcal{C})=\int_{\mathcal{C}} P(c(s))^{-2}+(P(c(s))-1)^{2} \mathrm{~d} s,
$$

where

$$
P\left(x_{1}, x_{2}\right)=D_{\overrightarrow{\mathbf{n}}} G\left(x_{1}, x_{2}\right) \quad \text { and } \quad \overrightarrow{\mathbf{n}}=\nabla G\left(x_{1}, x_{2}\right)\left\|\nabla G\left(x_{1}, x_{2}\right)\right\|^{-1},
$$

and $D_{\overrightarrow{\mathbf{n}}}$ is the directional derivative operator in the direction $\overrightarrow{\mathbf{n}}$. That is, we keep the directional derivative of $G(\mathbf{p})$ in each point of the segment $\mathcal{C}$ in the normalized gradient direction far away from zero and close to 1 (without loss of generality).

The evaluation of the (13) and its derivatives with respect to the coefficients $b_{\mathbf{i}}$ is similar to the evaluation of (9).

Lemma 2 If the point $\mathbf{b}_{0} \in \mathbb{R} \mathbb{P}^{d_{n}-5}$ represents an algebraic curve $\mathcal{C}$ which satisfies the conditions (1) and (2) of the Definition 1 with a singularity on $\mathcal{C}$, then the function $f_{\text {penalty }}(\mathcal{C})$ defined by $(13)$ has a singularity in the point $\mathbf{b}_{0}$ and

$$
\lim _{\mathbf{b} \rightarrow \mathbf{b}_{0}} f_{\text {penalty }}(\mathbf{b})=+\infty
$$

Proof: Let $\left\{\mathbf{b}_{i}\right\}_{i=1}^{\infty} \subset \mathbb{R} \mathbb{P}^{d_{n}-5}$ be a sequence of points representing regular algebraic curves as in (6), such that

$$
\lim _{i \rightarrow \infty} \mathbf{b}=\mathbf{b}_{0} .
$$

Let $\mathbf{c}_{i}(s)$ be the natural parameterizations of the curves in the neighborhood of $\mathbf{c}_{i}(0)$ and $\mathbf{c}_{i}(0) \rightarrow \mathbf{p}$, where $\mathbf{p}$ is a singularity on $\mathcal{C}$. Since the convergence of the curves $\mathbf{c}_{i}$ towards the singular limit curve $\mathcal{C}$ is uniform and $\nabla F$ is continuous, for some $\varepsilon>0$

$$
\lim _{i \rightarrow \infty} \int_{0}^{\varepsilon}\left\|\nabla F\left(\mathbf{c}_{i}(s)\right)\right\|^{-2} \mathrm{~d} s=+\infty
$$

and (15) follows easily.

Consequently, the modification of the objective function leads to

$$
f(\mathbf{b})=f_{\text {bend }}(\mathbf{b})+\alpha_{\text {penalty }} f_{\text {penalty }}(\mathbf{b})
$$

with a non-negative weight $\alpha_{\text {penalty }}$. 


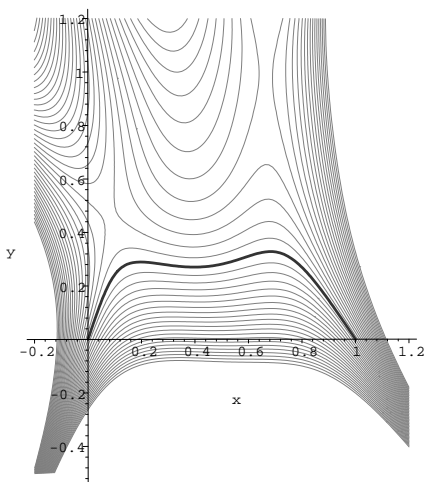

original curve

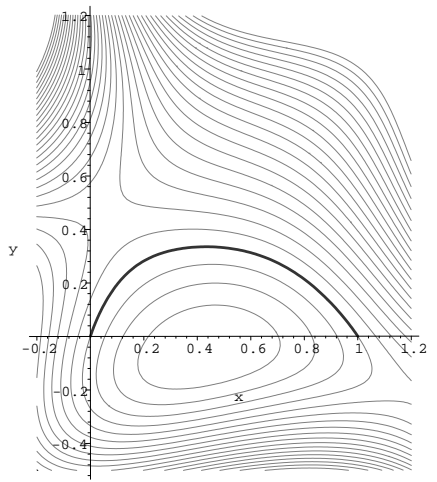

$\alpha=1.5$

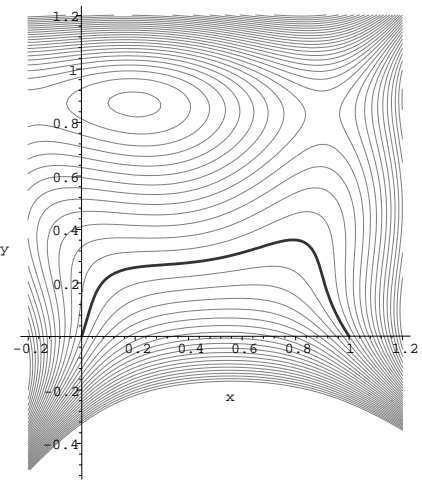

$\alpha=50$

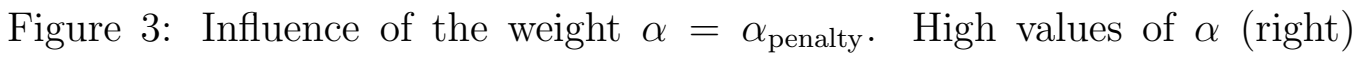
keep singularities (more precisely: vanishing gradients) farther away from the segment $\mathcal{C}$. However, the energy of the curve is increased in this case. Note that the range and distance of the level curves (algebraic offsets) are identical.

Corollary 1 The algebraic curve minimizing (18) has no singularities along the considered curve segment.

Clearly, the influence of the penalty function can be controlled by the weight. This is demonstrated by Figure 3. We started the optimization with the curve segment shown on the left. The middle and right figures have been obtained after two steps of the iteration with weights $\alpha_{\text {penalty }} \in\{1.5,50.0\}$. Note the different shape of the level curves (algebraic offsets).

The example in the Figure 4 illustrates the fairing of a quartic algebraic curve. We fair the curve in Figure 4 , (a) which has a bending energy $f_{\text {bend }}=$ 67.92. Figure 4, (b) shows the result of the fairing with weight $\alpha_{\text {penalty }}=0.5$. The bending energy decreases to $f_{\text {bend }}=4.1$. This is to be compared with the quartic polynomial approximation ${ }^{2}$ of the elastic curve in Figure 4, (c) with bending energy value along the considered segment 5.41.

\footnotetext{
${ }^{2}$ It has been generated with the help of numerical optimization where the cubic Hermite interpolant served as an initial value.
} 


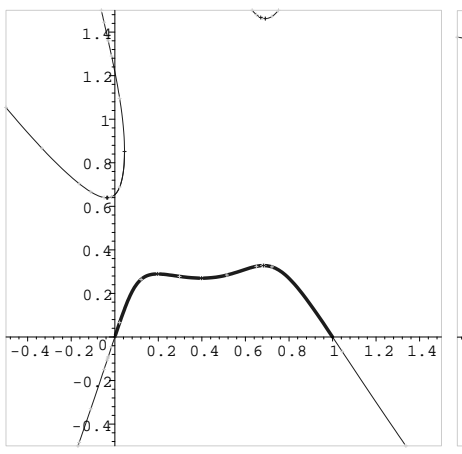

(a)

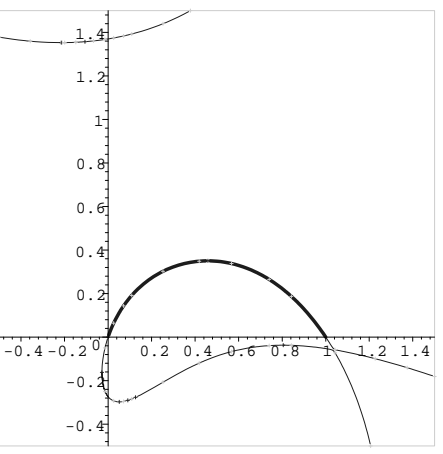

(b)

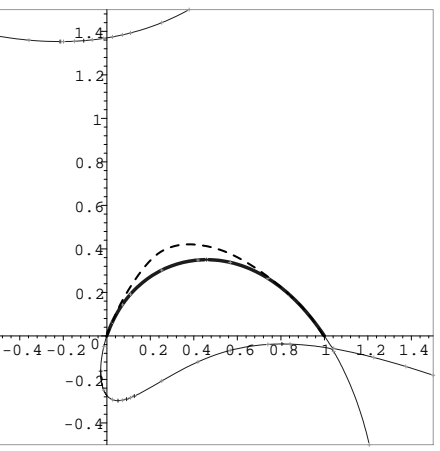

(c)

Figure 4: Fairing an algebraic curve segment using the penalty function (a): initial curve, (b), optimized algebraic curve, (c) dashed line curve - degree 4 polynomial which minimizes the bending energy.

\section{Algebraic Curve Shaping}

Variational modeling comprises the shape optimization of objects subject to geometric constraints. We use the numerical technique to solve the following problem: Find an algebraic curve segment, such that

1. it interpolates given points and tangents at them,

2. the curve is as fair as possible (cf. Section 2.2), without singularities,

3. the curve is pulled toward chosen fixed point $\mathbf{p}$.

This leads to the optimization problem presented in Section 3, with an additional term responsible for the condition 3 .

Let $\mathbf{p}=\left[\bar{x}_{1}, \bar{x}_{2}\right]^{\top}$. As a naive idea, the additional term of the objective function could be chosen an expression $G\left(\bar{x}_{1}, \bar{x}_{2}\right)^{2}$. This is called the algebraic distance of a point $\mathbf{p}$ from the curve $G=0$. Unfortunately, this approach does not work in those cases, where another branch of the algebraic curve is present in a neighborhood of the point $\mathbf{p}$ (see e.g. Figure 5, right). Clearly, in this case the algebraic distance might be measured with respect to the wrong branch.

As a more geometrically oriented approach we use, is the squared distance of $\mathbf{p}$ from the segment $\mathcal{C}$,

$$
f_{\text {pull }}(\mathcal{C})=\left\|\left(\mathbf{c}\left(s_{\mathbf{p}}\right)-\mathbf{p}\right)\right\|^{2}
$$

where the point $\mathbf{c}\left(s_{\mathbf{p}}\right)$ is the footpoint from the point $\mathbf{p}$ with the minimum 


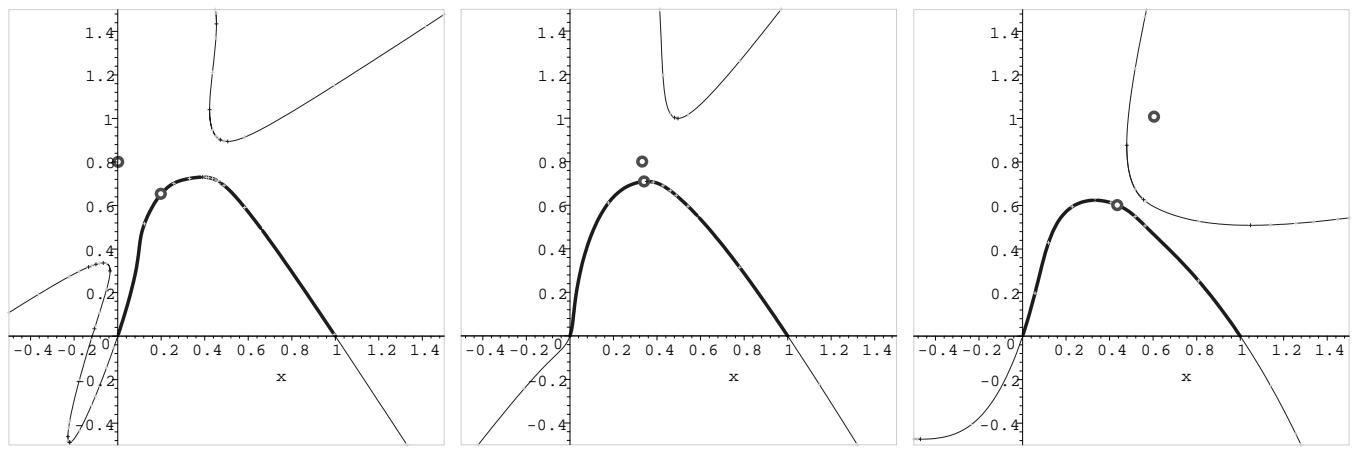

Figure 5: The pulling of the algebraic curve segment towards a point

distance property

$$
\left\|\mathbf{c}\left(s_{\mathbf{p}}\right)-\mathbf{p}\right\|=\min _{\substack{s \in I \\ \mathbf{c}(s) \text { is footpoint from } \mathbf{p}}}\|\mathbf{c}(s)-\mathbf{p}\| .
$$

We assume, that the footpoint exists, otherwise we take either the closest point on the segment $\mathcal{C}$ or the point which forms a minimal angle of vectors $\mathbf{p}-\mathbf{c}(s)$ and the $\nabla G(c(s))$. Hence, the final form of the objective function is

$$
f(\mathbf{b})=f_{\text {bend }}(\mathbf{b})+\alpha_{\text {penalty }} f_{\text {penalty }}(\mathbf{b})+\alpha_{\text {pull }} f_{\text {pull }}(\mathbf{b}) .
$$

The examples of such optimization for various points $\mathbf{p}$ can be seen in the Figure 5. The initial curve segment (see Figure 4, left) was pulled toward three different points $[0,0.8]$ (left), $\left[\frac{1}{3}, 0.8\right]$ (middle) and [0.6, 0.8] (right). The final bending energy resp. squared distance of the point $\mathbf{p}$ to the curve segment rich the values $f_{\text {bend }}=10.04, f_{\text {pull }}=0.06$ (left), $f_{\text {pull }}=0.001$, $f_{\text {bend }}=12.44$ (middle) and $f_{\text {bend }}=8.44, f_{\text {pull }}=0.18$ (right). The corresponding weights in the objective function $(20)$ are $\left(\alpha_{\text {penalty }}, \alpha_{\text {pull }}\right)=(0.00001,350)$ (left),$\left(\alpha_{\text {penalty }}, \alpha_{\text {pull }}\right)=(0.0001,300)($ middle $)$ and $\left(\alpha_{\text {penalty }}, \alpha_{\text {pull }}\right)=(0.03,80)$ (right).

Clearly, the results of the fairing process can be further controlled by the ratio of the parameters $1: \alpha_{\text {penalty }}: \alpha_{\text {pull }}$ to balance the curvature and the closeness of the curve to the chosen point $\mathbf{p}$. We can try to pull the curve as close to the point $\mathbf{p}$ as possible with raising the weight $\alpha_{\text {pull }}$. The bending energy usually raises considerably as the curve gets closer to the point $\mathbf{p}$.

Experiments with different weights in the objective function are shown in Figure 6. The same initial segment as in Figure 5 was used in both cases. For 

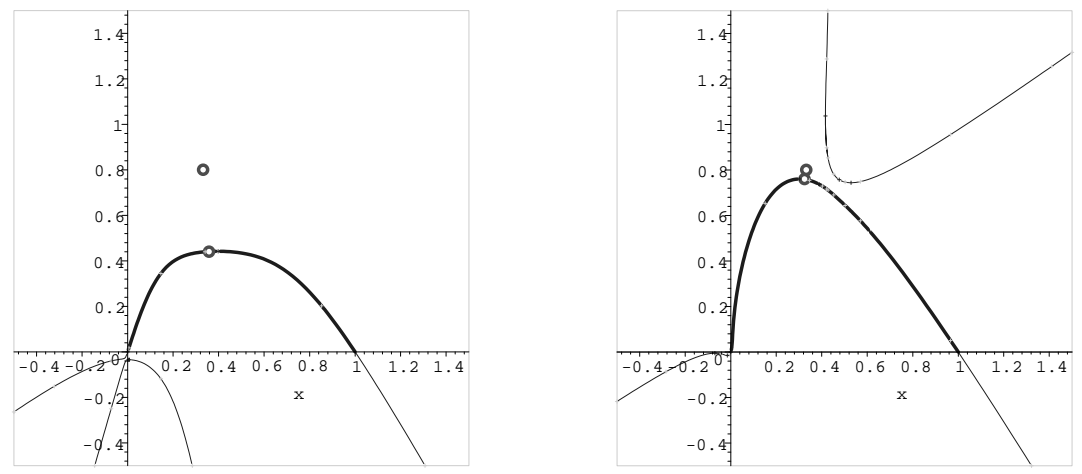

Figure 6: Adjusting the pulling weight in the objective function. By increasing the pulling weight, the point is brought closer to the resulting curve.

the left figure, $\left(\alpha_{\text {penalty }}, \alpha_{\text {pull }}\right)=(0.05,100)$, the square of the final distance increased to $f_{\text {pull }}=0.06$ and the bending energy decreased to $f_{\text {bend }}=9.73$. For the right figure, $\left(\alpha_{\text {penalty }}, \alpha_{\text {pull }}\right)=(0.0001,750)$, the final bending energy is $f_{\text {bend }}=12.44$ and $f_{\text {pull }}=0.001$.

\section{Conclusions and Future Work}

We have presented a method for variational design at algebraic curves. By applying our method to an algebraic curve, it is possible to get a fair algebraic curve segment without singular points and minimal bending energy. Moreover, the algorithm can be extended by additional tools, such as pulling, for designing algebraic segments. All tools are configurable by parameters with intuitive meaning. As a novel approach, we demonstrated, that regularity of the curve can be guaranteed by suitable modification of the objective function (penalty function). The alternative technique is the use of discriminating families (see $[1,13,14]$ ).

There are several possible extensions. Firstly, the method could be applied to algebraic splines (i.e. piecewise algebraic curves). In addition to applying fairing process to the polynomial pieces, one needs to find an appropriate way of minimizing curvature discontinuities, which occur - in the $G^{1}$ case - at the boundaries of the polynomial segments. This seems to be a non-trivial task.

Another challenging problem is the extension to the case of algebraic surfaces, where the minimization itself is less well understood. The existence 
of the minimum for Willmore-type energies for certain classes of surfaces is known (see e.g. [10]). In the surface case one has to deal with more complicated domains, which causes many technical difficulties.

\section{References}

[1] C.L. Bajaj and G. Xu. Regular algebraic curve segments (III) - applications in interactive design and data fitting. Comput. Aided Geom. Des., 18(3):149-173, 2001.

[2] Guido Brunnett, Hans Hagen, and Paolo Santarelli. Variational design of curves and surfaces. Surveys Math. Indust., 3(1):1-27, 1993.

[3] Guido H. Brunnett. Properties of minimal-energy splines. In Hagen, Hans (ed.), Curve and surface design, SIAM, Philadelphia, pages 3-22. 1992.

[4] Pavel Chalmovianský and Bert Jüttler. Fairness criteria for algebraic curves. Technical report, Johannes Kepler University Linz, Spezialforschungsbereich SFB013, March 2003.

[5] Günther Greiner. Surface construction based on variational principles. In Laurent, Pierre-Jean (ed.) et al., Wavelets, images, and surface fitting. A $K$ Peters Wellesley, pages 277-286. 1994.

[6] H. Hagen. Variational principles in curve and surface design. In Fisher, $R$. B. (ed.), Design and application of curves and surfaces. (Mathematics of surfaces V.) Clarendon Press Oxford, pages 169-190. 1994.

[7] Emery Jou and Weimin Han. Minimal-energy splines with various end constraints. In Hagen, Hans (ed.), Curve and surface design. SIAM Philadelphia, pages 23-40. 1992.

[8] B. Jüttler. Least-squares fitting of algebraic spline curves via normal vector estimation. In R. Cipolla and R.R. Martin, editors, The Mathematics of surfaces IX., pages 263-280. Clarendon Press, Oxford, 2000.

[9] Bert Jüttler and Alf Felis. Least-squares fitting of algebraic spline surfaces. Adv. Comput. Math., 17(1-2):135-152, 2002.

[10] Andreas Kipp and Ulrich Reif. On the existence of solutions to non-parametric fairing problems. J. Math. Anal. Appl., 238(2):540-550, 1999.

[11] Thomas W. Sederberg. Planar piecewise algebraic curves. Comput. Aided Geom. Des., 1:241-255, 1984.

[12] Osher Stanley and Fedkiw Ronald. Level Set Methods and Dynamic Implicit Surfaces. Springer, Heidelberg, 2003.

[13] G. Xu, C.L. Bajaj, and C.I. Chu. Regular algebraic curve segments. II: Interpolation and approximation. Comput. Aided Geom. Des., 17(6):503-519, 2000.

[14] G. Xu, C.L. Bajaj, and W. Xue. Regular algebraic curve segments. I: Definitions and characteristics. Comput. Aided Geom. Des., 17(6):485-501, 2000. 